\title{
Control and Simulation of Ventilation System of Residential Buildings
}

\author{
Zhaowen Zhang \\ Institute of Oceanographic Instrumentation, Shandong Academy of Sciences, Qingdao, Shandong, \\ 266071, China
}

\begin{abstract}
According to the basic control law of HVAC automatic control as well and the fuzzy control theory, it can determine the residential control strategy. Besides, it can simulate the ventilation effect of human breathing, cooking and other different disturbance interference effects by using Simulink simulation software of MATLAB, so as to calculate the fresh air volume maintaining the indoor $\mathrm{CO}_{2}$ concentration at $850 \mathrm{ppm}$ as well as the needed time. And then, it can further prove that when indoor ventilation is 0.5 times per hour, the indoor air quality cannot be guaranteed. Therefore, we cannot intuitively think that the greater amount of ventilation, the better removal effect of the air pollutant will be. The study also prove the necessity to use automatic control means to improve the residential indoor environment, show that the PID fuzzy control method can guarantee the overshoot control in a small amount with stability and high precision with high speed.
\end{abstract}

Keywords: HVAC, MATLAB, Ventilation System, Buildings

\section{Introduction}

Since China stepped into the twenty-first century our national economy has got steady and rapid development, people can fully enjoy the achievements brought by the rapid development of the national economy, gradually people produced a deeper understanding to the life, work and living environment, thus the requirement on the quality of indoor air environment is increasingly high. As for those people, who almost spend about $80 \%$ of their whole life time in the room, whether the indoor air environment is good or bad, comfortable or not has significant meaning on health.Especially in residential buildings, both kitchen and bathroom are the important pollution source of the indoor environment, on one hand, from the functional perspective, during the process that residents use to produce the odor, when oil fume is burning, $\mathrm{SO}_{2}, \mathrm{CO}, \mathrm{NO}_{2}, \mathrm{CO}_{2}$ and other harmful gases will be produced at the same time, which can endanger people's health; on the other hand, from the perspective of structure characteristics, the equipment from the building materials used, plate, floor tiles material can produce hazardous substances as well as noises, which can have a certain influence on people's life[1-3].

When $\mathrm{CO}_{2}$ concentration sensor and air volume regulator can be applied to the residential buildings[4], the indoor air quality can be improved by controlling the kitchen ventilation mode. This chapter, we will study on the basic principle of HVAC control, selecting the control strategy of residential ventilation, using Simulink simulation software to simulate the change trend in different disturbance under the concentration of $\mathrm{CO}_{2}$, with the tendency of the required air volume as well as the stable time, so as to ensure fast and stable regulation mode. 


\section{Automatic Control Principle of HVAC System}

The basic concept of automatic control refers to using one or more physical quantities (such as voltage, current, speed, position, temperature, fluid, chemical composition) of the control device object (such as machinery, equipment or production process) to operate or change automatically according to the predetermined rules without the direct participation of human.

The working state of the controlled object's automatic control system can be called as automatic control system. The function and composition of the automatic control system are various, the composition structure can be simple and direct which can also be complicated and variable. The system can control a single physical quantity, which can also be variables; in addition, it can be a specific engineering project, such as an enterprise's production managing process, which can also be an abstract system.

\section{The Composition of HVAC Automatic Control System}

The automatic control system is composed of two parts, one part is the automatic device that can realize the automation of automatic control, including the transmitter, controller and actuator and so on, the other part is the required controlled object. The controlled object generally refers to the equipment, machinery or production process that needs to achieve automatic control; while the controlling device refers to the overall equipment that can control the controlled object. These components can be integrated into the construction of air conditioning and heating equipment, which can be further introduced:

(1) The Controlled Object

In HVAC major, a variety of air-conditioned rooms, heat transfer equipment, refrigeration equipment, heating boilers, heating and gas network can be regarded as the controlled objects. Besides, pressure, temperature, humidity, wind speed and other parameters can also be regarded as the controlled objects in some large refrigeration units or air conditioning heating rooms. It can be seen that the controlled object is not necessarily the whole device in equipment, and there is not only one controlled object in a device.

The controlled object is expected to be maintained at a fixed value or set range, in the actual process, the difference between the measured value and the set value of the controlled object can be called as deviation. The factors that can make the variables of the controlled object deviate from the set value can be called as interference, while the variables that are used to overcome the influence of these disturbances can be called as the adjusting parameters.

(2) Measuring Transmitter

The measuring transmitter is composed by transmitter and sensor. It can transfer the instrument for measuring the signal into signal behind the instrument into the transmitter; while measuring sensor is used to sense the changes of process parameters detecting element, the way of expressing the output signal with the instrument behind is quite different. The sensor in the HVAC specialty is including lithium chloride resistance type humidity sensor, resistance type remote pressure gauge, infrared gas analyzer and electromagnetic flowmeter[5].

(3) Regulator

Regulator can be responsible for comparing the signal which the transmitter conversed with the set value that should be maintained, calculating the deviation value, according to this deviation value, it can send out the conversion signal to the actuator in accordance with the provisions of the operational rules. 


\section{(4) Actuator}

The output signal of the regulator can realize the variable control of the controlled object by the adjustment of the actuator. The actuators can be divided into two types, namely, electric actuators and pneumatic actuators, the actuators which are commonly used in construction equipment, such as electric control valve, pneumatic control valve, electric air valve, three way control valve, straight adjustment valve, electric valve positioner.

\section{The Expression of Automatic Control System of HVAC}

The principle of automatic control system commonly used block diagram to express. The block diagram is composed of a block, signal line, input signal, output signal as well as comparison section, which can make the structure of a control system and the signal be displayed visually in the block diagram. As shown in Fig. 1, it is a closed loop feedback control system, each block can represent a link in the control system. In this study, when there is a disturbance personnel interference signal into the controlled object $\mathrm{CO}_{2}$ concentration sensor, the output variable $Y$ of the controlled object can input to the detection device, transmitting the output signal into $Z$, so as to enter the comparison organization,comparing with $\mathrm{CO}_{2}$, which is hoped to keep the concentration value so that it can get the error signal $e$. After entering the the controller according to certain rules, outputting the control signal to the actuator of kitchen exhaust system, regulator can change the size of the exhausting air after receiving the signal, so as to control variables and reduce the impact cause by the interference.

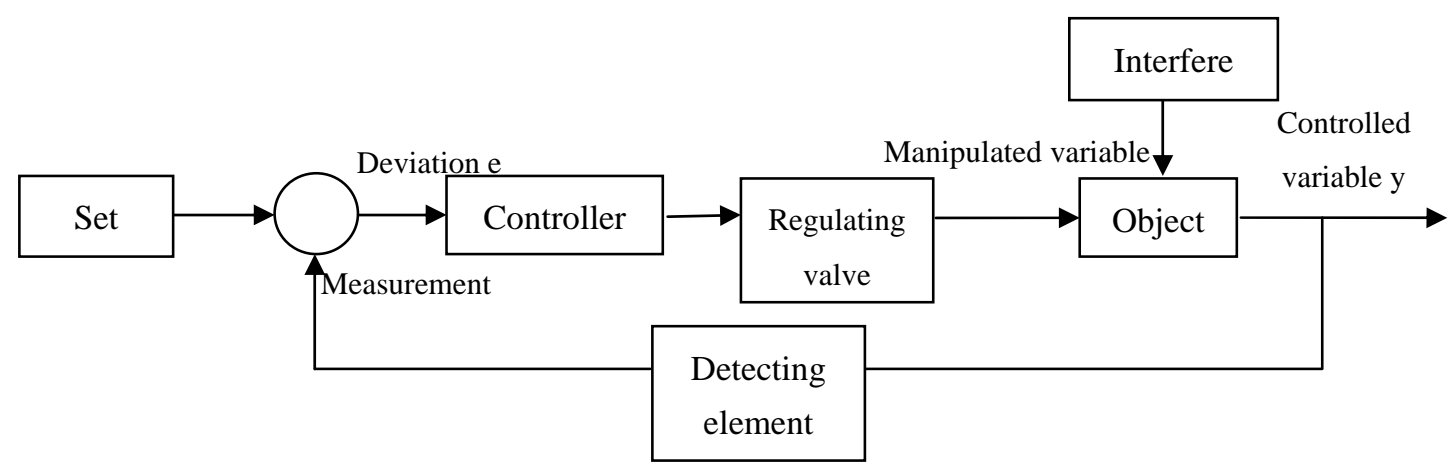

Fig. 1 Block diagram of automatic control system

\section{Selection and Determination of Basic Control Law}

\section{Proportional Control Law}

Proportional (P) control law can be fully in accordance with the control variables and the size and direction of the deviation of the output value to send out the output signal, which can be proportional with the deviation value, there is a corresponding relationship between the position of the valve and the deviation. In the air conditioning system, so as to enlarge the temperature change of the controlled room by using proportional magnification coefficient or the interference signal of the load change, starting the air conditioning refrigeration units through adjusting the parameters of the flow and pressure as well as other parameters.

The main disadvantage of the proportional control law is that the residual error exists in the control process. The residual reason has two aspects: one is when the load changes greatly, in order to compensate the load changes, the required valve opening change will increase, which can result in large deviation of the control law, producing large residual error; the other is when the required control system lagging is large, which also does not allow the tuning of the proportion to be small, 
the residual error will be large. Therefore, as for the object with lager pure lagging time and smaller time constant, and the lager enlarged coefficient in the application of proportional control, the requirement on the adjustment of the proportion is quite larger, this kind of operation will bring the price that is to increase lager difference, the system will lost its effectiveness, the effect of control is not so completely.

$P$ control law is suitable for the system with small load change, large time constant is larger, while pure lagging is not too large, with the controlled variables that can have difference. The object range of the scale of the proportion is $30 \%-70 \%$, while the range of temperature object is $20 \%-60 \%$ and so on.

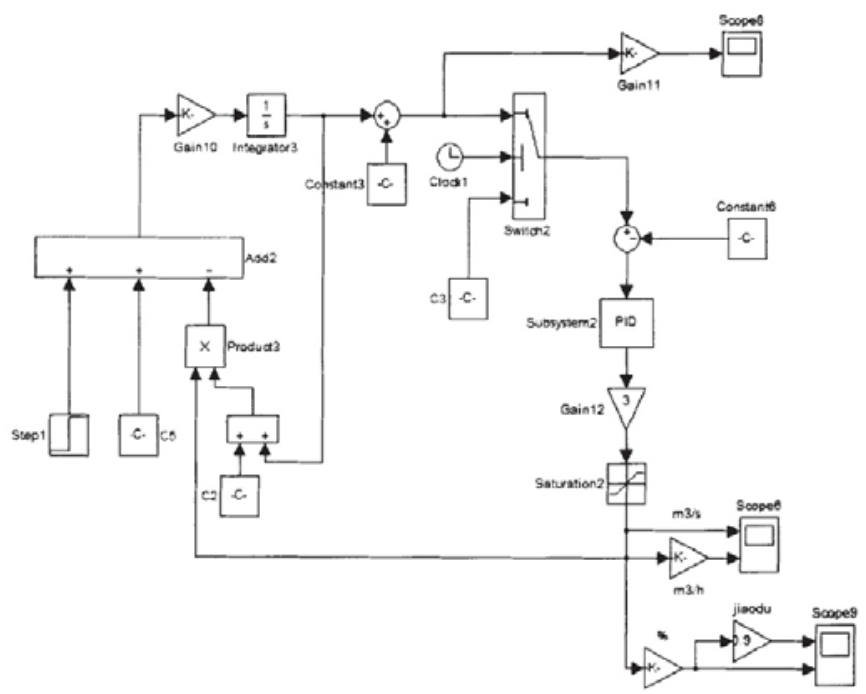

Fig. 2 Simulation Control Diagram in Simulink

Editing Image Language in MATLAB:

shh=get( 0 ,'ShowHiddenHandles');

set( 0 ,'ShowHiddenHandles','On')

set(gcf,'menubar','figure')

set(gcf, 'CloseRequestFcn','closereq')

set(gcf, 'DefaultLineClipping','off')

set(0,'ShowHiddenHandles',shh)

\section{Analysis on Simulation Results}

Under the initial state that three people is the the room originally, according to the calculation, when the concentration of $\mathrm{CO}_{2}$ in $2965 \mathrm{~s}(0.82 \mathrm{~h})$ is accumulated to $1000 \mathrm{ppm}$, it can start fan at this moment, exhausting respectively by different amount of exhausting air, after reaching to a steady state, increasing the different values of disturbance at different times, minus the set the concentration value, so as to calculate out the difference as the step interference signal, which can be controlled by PID system. We can make comparison and analysis on the different working conditions together, which has added the same amount of disturbance, so as to have discussion with the result of the rest of the simulation respectively. 


\section{Reference}

[1] Pavlovas, Vitalijus. "Demand controlled ventilation: A case study for existing Swedish multifamily buildings." Energy and buildings 36.10 (2004): 1029-1034.

[2] Dorer, V., R. Weber, and A. Weber. "Performance assessment of fuel cell micro-cogeneration systems for residential buildings." Energy and Buildings37.11 (2005): 1132-1146.

[3] Lihua, Wang. "Design of Coconut Feeding Machine Based on Motion Controller." Journal of Applied Science and Engineering Innovation 2.5 (2015): 160-162.

[4] Du, Yuyang, et al. "Implementation and Design of Structure of Multilevel Secure Database System." Journal of Applied Science and Engineering Innovation 2.6 (2015): 220-222.

[5] Phillips, E. G., et al. "A model to compare freezing control strategies for residential air-to-air heat recovery ventilators." ASHRAE Transactions (American Society of Heating, Refrigerating and Air-Conditioning Engineers);(USA) 95.CONF-890609-- (1989). 